\title{
The Mean Curvature of the Influence Surface of Wave Equation With Sources on a Moving Surface
}

\author{
F. Farassat \\ Mail Stop 461, NASA Langley Research Center, Hampton, Virginia \\ 23681-2199, USA \\ Mark Farris \\ Midwestern State University, Wichita Falls, Texas, USA
}

Journal Mathematical Methods in the Applied Sciences

Volume 22, 1999, 1485-1503 


\title{
The Mean Curvature of the Influence Surface of Wave Equation With Sources on a Moving Surface
}

\author{
F. Farassat \\ Mail Stop 461, NASA Langley Research Center, Hampton, Virginia 23681-2199, \\ USA
}

Mark Farris

Midwestern State University, Wichita Falls, Texas, USA

MSC (MOS) Classification Numbers: 35L05, 53B50, 76J20, 76Q05, 46F10

Summary

The mean curvature of the influence surface of the space-time point $(\boldsymbol{x}, t)$ appears in linear supersonic propeller noise theory and in the Kirchhoff formula for a supersonic surface. Both these problems are governed by the linear wave equation with sources on a moving surface. The influence surface is also called the $\Sigma$ - surface in the aeroacoustic literature. This surface is the locus, in a frame fixed to the quiescent medium, of all the points of a radiating surface $f(\boldsymbol{x}, t)=0$ whose acoustic signals arrive simultaneously to an observer at position $\boldsymbol{x}$ and at the time $t$. Mathematically, the $\Sigma$-surface is produced by the intersection of the characteristic conoid of the space-time point $(x, t)$ and the moving surface. In this paper, we derive the expression for the local mean curvature of the $\Sigma$ - surface of the space-time point $(\boldsymbol{x}, t)$ for a moving rigid or deformable surface $f(\boldsymbol{x}, t)=0$. This expression is a complicated function of the geometric and kinematic parameters of the surface $f(\boldsymbol{x}, t)=0$. Using the results of this paper, the solution of the governing wave equation of high speed propeller noise radiation as well as the Kirchhoff formula for a supersonic surface can be written as very compact analytic expressions. 


\subsection{Introduction}

The prediction of the noise high speed helicopter rotors and propellers is an important aeroacoustic problem. Until recently there have been two approaches in wide practice to attack this problem. The first is based on the Ffowcs Williams-Hawkings (FW-H) equation [1] and the second is based on the Kirchhoff method. The Kirchhoff method was suggested first by Hawkings for aeroacoustic applications [2]. There are now a large number of publications on the use of FW-H equation. In general, these applications use this equation with surface sources specified on an impenetrable surface such as a blade surface. See the many references in various chapters of Hubbard [3] and Goldstein [4]. A good review of the applications of the Kirchhoff method is by Lyrintzis [5]. Ffowcs Williams also proposed to use FW-H equation with the data specified on a penetrable surface [6, Chap.11, Sec. 10]. This was carried out for high speed helicopter rotor noise prediction by $\mathrm{Di}$ Francescantonio [7] and, independently by Brentner and Farassat [8]. This new method essentially brings the two approaches together and appears to be superior to the earlier approaches [8]. The point of relevance to the present paper is that in all these methods, one has to solve the linear wave equation with sources on a moving surface. We will address the difficult problem of the solution of the linear wave equation with supersonic surface sources here. As in all of our previous works, we will solve this problem in the time domain.

The mean curvature $H_{F}$ of the influence surface of the space-time point $(\boldsymbol{x}, t)$ appears in linear supersonic propeller noise theory and the Kirchhoff formula for a supersonically moving surface [9-11]. We refer to the influence surface as the $\Sigma$ - surface in this paper to be consistent with our earlier publications on the subject of linear wave propagation. Assuming that the propeller blade or the Kirchhoff surface is given by the moving surface $f(x, t)=0$, the $\Sigma$ - surface is constructed by the intersection of the characteristic conoid of the space-time point $(\boldsymbol{x}, t)$ and the moving surface $f=0$. This construction is explained in the present paper in detail. The $\Sigma$ - surface is, thus, the locus, in the frame fixed to the quiescent medium, of all the points on the surface $f=0$ whose acoustic signals arrive simultaneously at $\boldsymbol{x}$ at the observer time $t$. Therefore, the $\Sigma$-surface describes the domain of 
dependence of the space-time point $(x, t)$ in the frame fixed to the quiescent medium.

In our work on linear supersonic propeller noise prediction in the time domain, many curvature terms of the blade surface appear in the theoretical formulation [9-11]. Our experience with numerical evaluation of propeller noise indicates that the curvature terms can contribute significantly to the predicted noise. For this reason, as well as to obtain the Kirchhoff formula for a supersonically moving surface, we give the detailed derivation of the analytic expression for the mean curvature of the $\Sigma$ - surface. The derivation requires the knowledge of classical differential geometry and tensor analysis [12-14]. In practice, the geometry and the kinematics of the moving surface $f=0$ are known. The mean curvature $H_{F}$ of the $\Sigma$ - surface must be described in terms of the known geometric and kinematic parameters of the surface $f=0$. This is achieved by first defining a local time dependent coordinate system on the $\Sigma$ - surface in terms of the local coordinate system on the surface $f=0$. The coefficients of the first and the second fundamental forms of the $\Sigma$ - surface are then written in terms of the known local parameters of the surface $f=0$. From these coefficients we construct the expression for the mean curvature $H_{F}$.

In section 2, we explain how the mean curvature of the $\Sigma$ - surface appears in supersonic propeller noise theory and the Kirchhoff formula for moving surfaces. In section 3 we describe the construction of the $\Sigma$ - surface and how to visualize this surface for a given observer space-time point $(x, t)$ when the motion and the geometry of $f=0$ are specified. In Section 4, which is the main part of this paper, we give the detailed derivation of the mean curvature $H_{F}$ assuming that the surface $f=0$ is rigid. In a subsection, we present a brief derivation of $H_{F}$ for a deformable surface. Discussion of the main results and concluding remarks are in Section 5. Here we discuss the validity of the expression for the mean curvature and the possibility of singularity formation in the acoustic field of a supersonically moving surface.

The main mathematical reference for this paper is [15] where all the rules of manipulation of multidimensional generalized functions used here are pre- 
sented. More details on differential geometry and Green's function solution of wave equation with sources on a moving surface are presented in a NASA Technical Memorandum [16]. We use both vector and tensor notations in the present paper where appropriate. The summation convention is also used here.

\subsection{How the Mean Curvature of the $\Sigma$-Surface Appears in Applications}

In this section, we briefly explain how the mean curvature $H_{F}$ appears in two problems of acoustics. The first is the supersonic propeller noise prediction problem and the second is the derivation of the Kirchhoff formula for a supersonically moving surface. Both these problems are governed by the linear wave equation with sources on a moving surface.

\subsection{The Supersonic Propeller Noise Problem}

Consider a supersonic propeller whose blades are described by $f(\boldsymbol{x}, t)=0, f>0$ outside the body. We assume that $f(\boldsymbol{x}, t)=0$ is so defined that $\nabla f=\boldsymbol{n}$, where $\boldsymbol{n}$ is the local unit outward normal to this surface. This can always be accomplished. Using the acoustic analogy of Lighthill, the governing equation is the Ffowcs Williams-Hawkings (FW-H) equation. For the purpose of discussion here, we are only interested in the surface source terms of this equation. The governing linear wave equation is, thus, the following:

$$
\square^{2} p^{\prime}=\frac{\partial}{\partial t}\left[\rho_{0} v_{n} \delta(f)\right]-\frac{\partial}{\partial x_{i}}\left[p n_{i} \delta(f)\right]
$$

where $p^{\prime}=\rho_{0} c^{2}, \rho_{0}$ and $c$ are the density and speed of sound in the undisturbed medium, respectively, $v_{n}$ is the local normal velocity on the blade and $p$ is the (gage) pressure on the blade. The Dirac delta function is denoted $\delta(f)$. We have assumed inviscid fluid medium here and have neglected the viscous forces on the fluid at the blade surface. 
In practice, one usually divides the propeller blade surface into panels and then sums up the noise produced by each panel [11]. For panels where the Doppler factor $\left|1-M_{r}\right|$ is not small, a noise prediction formula based on the Green's function solution of Eq.(1) with the Doppler factor (e.g., Formulation 1A of Langley $[17,18])$ can be used. Here $M_{r}$ is the local Mach number of the surface in the radiation direction based on the speed of sound in the undisturbed medium. The Doppler factor appears in the denominator of this solution of Eq.(1) and, therefore, it is also referred to as the Doppler singularity. For supersonic propellers, the Doppler factor can be small or zero for many panels on the blade for a given observer position and time. In that case, another solution of the FW-H equation without the Doppler singularity must be used for noise prediction $[10,11]$. We present a sketch of the derivation of this solution below.

We assume that the blade surface $f=0$ is smooth. This is a simplifying assumption which is not satisfied in practice, e.g., at the trailing edge of the blade, but does not concern us here. See references $[10,11,16]$ for the general case. Using the rules of generalized differentiation $[15,16,19,20]$, the derivatives on the right side Eq.(1) are taken explicitly giving the following result:

$$
\begin{gathered}
\frac{\partial}{\partial t}\left[\rho_{0} v_{n} \delta(f)\right]=\rho_{0} \dot{v}_{n} \delta(f)-\rho_{0} \underset{\sim}{v_{n}^{2}} \delta^{\prime}(f), \\
\frac{\partial}{\partial x_{i}}\left[p n_{i} \delta(f)\right]=-2 H_{f} p \delta(f)+\underset{\sim}{p} \delta^{\prime}(f)
\end{gathered}
$$

where $H_{f}$ is the local mean curvature of the blade surface $f=0$ and a tilde under a variable, such as $\underset{\sim}{p}$, denotes the restriction of the variable to the surface $f=0$. See references $[15,16]$ for the concept of restriction. As mentioned in references $[9,15,16]$, the purpose of restriction of a variable to $f=0$ is to reduce algebraic manipulations as well as obtaining analytic expressions in the simplest possible form. Using Eqs.(2) and (3) in Eq.(1), we get:

$$
\square^{2} p^{\prime}=\left(\rho_{0} \dot{v}_{n}+2 H_{f} p\right) \delta(f)-\left(\underset{\sim}{p}+\rho_{0} \underset{\sim}{\underset{v}{2}}\right) \delta^{\prime}(f)
$$

We will give the solution of the wave equation with these types of inhomogeneous source terms after the following discussion on the Kirchhoff formula 
for a supersonically moving surface. We mention here that it is the second term on the right of Eq.(4) involving $\delta^{\prime}(f)$ which will give an integrand depending on the mean curvature of the $\Sigma$ - surface when the Green's function solution of Eq.(4) is sought.

\subsection{The Kirchhoff Formula For a Supersonically Moving Surface}

The need for the development of the Kirchhoff formula for a supersonically moving surface appears in the prediction of the high speed rotor noise of a helicopter. Even though the conventional rotor blades travel at subsonic speed, the blade tip shock can move at supersonic speed. One possible method of prediction of high speed rotor noise is using the Kirchhoff formula for a moving surface. We will call the surface on which the data are specified the Kirchhoff surface. In practice one specifies the data on the Kirchhoff surface from high resolution unsteady aerodynamic calculations. Kirchhoff formula based methods are showing great promise in aeroacoustics [5].

Let the supersonically moving Kirchhoff surface be described by the equation $f(\boldsymbol{x}, t)=0, f>0$ outside the surface and $|\nabla f|=\boldsymbol{n}$. We assume again that $f=0$ is a smooth surface. In practice, this assumption is not satisfied because it is often more convenient to use a Kirchhoff surface with edges. References [9] and [16] give the general result which applies to piecewise smooth surface. Let $p^{\prime}$ stand for the acoustic pressure satisfying the linear wave equation in the region outside the surface $f=0$. The governing equation for deriving the Kirchhoff formula is obtained by first extending $p^{\prime}$ to the entire space as follows:

$$
\tilde{p}^{\prime}=\left\{\begin{array}{lll}
p^{\prime} & f>0 & \text { (outside the surface) } \\
0 & f<0 & \text { (inside the surface) }
\end{array}\right.
$$

Then, $\tilde{p}^{\prime}$ has a discontinuity across the surface $f=0$. It is seen that in the entire three dimensional space, we have

$$
\square^{2} \tilde{p}^{\prime}=0
$$


where all the derivatives in the wave operator are ordinary derivatives. The generalized D'Alembertian of $\tilde{p}^{\prime}$ gives the following equation $[9,15,16,19,20]$ :

$$
\bar{\square}^{2} \tilde{p}^{\prime}=-\left(p_{n}+\frac{1}{c} M_{n} p_{t}\right) \delta(f)-\frac{1}{c} \frac{\partial}{\partial t}\left[M_{n} p \delta(f)\right]-\nabla \cdot[p \boldsymbol{n} \delta(f)]
$$

where the bar over the wave operator stands for generalized differentiation $[15,19,20]$. Note that in this paper we use a bar over a differentiation operator to signify generalized differentiation only if there is the possibility of confusion between the ordinary and the generalized derivatives of a function. For example, in Eqs.(1) and (4) we have not used a bar over the wave operator even though the derivatives in the wave operator are generalized derivatives. It is clear from the right sides of these equations that the wave operator can only be generalized D'Alembertian.

Taking the derivatives on the right side of the above equation explicitly, and using results similar to those of Eqs.(2) and (3), give the following governing equation for deriving the Kirchhoff formula for a supersonically moving surface:

$$
\begin{aligned}
\bar{\square}^{2} \tilde{p}^{\prime}= & -\left[p_{n}+\frac{1}{c} M_{n} p_{t}+\frac{1}{c}\left({\underset{\sim}{M}}_{n} \underset{\sim}{p}\right)_{t}-2 H_{f} p\right] \delta(f) \\
& -\left(1-{\underset{\sim}{M}}_{n}^{2}\right) \underset{\sim}{p} \delta^{\prime}(f)
\end{aligned}
$$

Here, as before, we have used a tilde under a variable to indicate the restriction of the variable to the surface $f=0$. Note that we can not drop the tildes in $\left(\underset{\sim}{M_{n}} \underset{\sim}{p}\right)$, where the subscript $t$ stands for partial differentiation with respect to time, since $\left(\underset{\sim}{M_{n}} \underset{\sim}{p}\right)_{t} \neq\left(M_{n} p\right)_{t}$ (see reference [15]). As in the case of Eq.(4), it is the second term on the right of Eq.(8) involving $\delta^{\prime}(f)$ which gives an integrand depending on the mean curvature of the $\Sigma$ - surface when the Green's function technique is applied to Eq.(8) to find the Kirchhoff formula for a supersonically moving surface.

Below we will show how one interprets the solution of the wave equation with sources involving $\delta(f)$ and $\delta^{\prime}(f)$. 


\subsection{The Solution of Wave Equation With Sources Involving $\delta(f)$ and $\delta^{\prime}(f)$}

We now give the solution to the following two wave equations $[15,20]$ :

$$
\begin{aligned}
\square^{2} \Phi & =q(x, t) \delta(f) \\
\square^{2} \Phi & =\underset{\sim}{q}(x, t) \delta^{\prime}(f)
\end{aligned}
$$

Let $F(\boldsymbol{y} ; \boldsymbol{x}, t)=f\left(\boldsymbol{y}, t-\frac{r}{c}\right)=[f(\boldsymbol{y}, \tau)]_{\text {ret }}$, where $(\boldsymbol{x}, t)$ and $(\boldsymbol{y}, \tau)$ are the observer and the source space-time variables, respectively. The subscript ret stands for the retarded time and $r=|\boldsymbol{x}-\boldsymbol{y}|$. The surface $F=0$ is the $\Sigma$ - surface of which more will be said in the next section. The solution of Eq.(9) is:

$$
4 \pi \Phi(\boldsymbol{x}, t)=\int_{F=0} \frac{1}{r}\left[\frac{q(\boldsymbol{y}, \tau)}{\Lambda}\right]_{r e t} d \Sigma
$$

where the parameter $\Lambda$ is defined below:

$$
\Lambda=\left(1+M_{n}^{2}-2 M_{n} \cos \theta\right)^{1 / 2}
$$

Here, $M_{n}$ is the local normal Mach number of the surface $f=0$ and $\theta$ is the angle between $\boldsymbol{n}$ and the radiation direction $\mathbf{r}=\boldsymbol{x}-\boldsymbol{y}$.

The solution of Eq.(10) is given by:

$$
\begin{aligned}
4 \pi \Phi(\boldsymbol{x}, t)=\int_{F=0}\{ & -\frac{\partial}{\partial N}\left[\frac{1}{r}\left(\frac{q(\boldsymbol{y}, \tau)}{\Lambda}\right)_{r e t}\right] \\
& \left.+\frac{2 H_{F}}{r}\left(\frac{q(\boldsymbol{y}, \tau)}{\Lambda}\right)_{r e t}\right\} \mathrm{d} \Sigma
\end{aligned}
$$

where $\partial / \partial N=N . \nabla$ and $N$ is the unit outward normal to the $\Sigma$ - surface. We will give this unit normal explicitly in section 4 . The mean curvature of 
the $\Sigma$ - surface, $H_{F}$, appears in the above equation. Therefore, it appears in solutions of Eqs.(4) and (8). Note that the mean curvature $H_{f}$ of the surface $f=0$ also appears in Eqs.(4) and (8). This curvature depends on geometry of the surface $f=0$ which is known. We will not write the solutions to equations (4) and (8) since we have given the complete solutions in a more general setting in our earlier papers $[9,10,15,16]$. However, note that the solutions of Eqs.(4) and (8) as given here are very simple and compact analytic expressions in contrast to what we have published earlier, e.g., in [11]. We will say more on what $\Sigma$ - surface is and how to visualize it.

\subsection{How To Construct and Visualize the $\Sigma$ - Surface}

As mentioned above the mathematical representation of the $\Sigma$-surface is given by the relation:

$$
F(\boldsymbol{y} ; \boldsymbol{x}, t)=f\left(\boldsymbol{y}, t-\frac{r}{c}\right)=[f(\boldsymbol{y}, \tau)]_{r e t}=0
$$

We give some facts and information about this surface and the way to construct and visualize it in space. The characteristic cone of the wave operator:

$$
\square^{2}=\frac{1}{c^{2}} \frac{\partial^{2}}{\partial \tau^{2}}-\frac{\partial^{2}}{\partial y_{i}^{2}}
$$

at the point $(\boldsymbol{x}, t)$ is given by the relation:

$$
g=\tau-t+\frac{|\boldsymbol{x}-\boldsymbol{y}|}{c}=\tau-t+\frac{r}{c}=0
$$

Note that the variables of the wave operator are $(y, \tau)$ which are also the variables of the equation of the characteristic cone. These variables are called the source (or the field) space-time variables. The vertex of the cone is at the point $(\boldsymbol{x}, t)$ which is kept fixed in the analysis below. The space-time variables $(\boldsymbol{x}, t)$ are known as the observer variables. Equation (16) describes a cone in space-time because if the point $(y, \tau)$ is on the surface $g=0$, then all 
points of the straight line joining this point to the point $(\boldsymbol{x}, t)$ are on $g=0$. This is the property of a cone. To prove this fact, note that if $(y, \tau)$ satisfies Eq.(16), then the point $\boldsymbol{A}=(\alpha \boldsymbol{y}+(1-\alpha) \boldsymbol{x}, \alpha \tau+(1-\alpha) t)$ where $\alpha$ is an arbitrary number, also satisfies Eq.(16). But the point $\boldsymbol{A}$ lies on the straight line joining $(\boldsymbol{x}, t)$ and $(\boldsymbol{y}, \tau)$ in the four dimensional space.

The wave operator in Eq.(15) has constant coefficients. Therefore, the characteristic cone and the characteristic conoid at any point coincide. Equation (16), thus, describes the characteristic conoid at the point $(x, t)$. Remember that $(\boldsymbol{x}, t)$ is fixed in our analysis and this point specifies the position of the vertex of the cone and the conoid. What is the surface $g=0$ ? Since it is impossible to visualize a surface in four dimensions, we must keep some variables fixed. Let us fix the source time variable $\tau$ in addition to $(\boldsymbol{x}, t)$. Then, the surface $g=0$, in variable $y$, is a sphere in three dimensional space with center at $\boldsymbol{x}$ and radius $c(t-\tau)$ for all time $\tau \leq t$. The radius of this sphere becomes zero at $\tau=t$. As the source time variable $\tau$ varies from $-\infty$ to $t$, the radius of the sphere shrinks at the speed of sound $c$ from $\infty$ to zero. This is why the characteristic cone $g=0$ of the wave equation is called the collapsing sphere when viewed dynamically as explained here. We will use this terminology in the present paper.

From the theory of hyperbolic partial differential equations $[21,22]$, the characteristic conoid at the point $(\boldsymbol{x}, t)$ determines the domain of dependence of this point on the initial and the boundary data. We can illustrate this schematically for the wave equation with constant coefficients in three space variables. The equation of the characteristic conoid is given by Eq.(16). Figure 1 shows this cone intersecting the three dimensional space, shown as planes, at various source time $\tau \leq t$. The domain of dependence of the point $(\boldsymbol{x}, t)$ is the circular region inside the cone where it intersects the three dimensional space at the given source time. Any data specified on this circular region influences the solution of the wave equation at $(\boldsymbol{x}, t)$. In the problems considered here, the data are specified on a moving surface, e.g. a propeller blade, and our wave equation has three space variables. How do we determine the domain of the dependence of the space-time point $(\boldsymbol{x}, t)$ in this case? 
The surface $f=0$ on which aerodynamic, kinematic or Kirchhoff data are specified, moves and possibly deforms in space. Since we have reserved the variables $(\boldsymbol{x}, t)$ for the observer, we now use the source variables for this surface, that is we write $f(\boldsymbol{y}, \tau)=0$. The influence surface, or the $\Sigma$ - surface associated with a given observer space-time variables $(\boldsymbol{x}, t)$ is obtained by the intersection the characteristic cone (conoid) of this point and the surface $f(\boldsymbol{y}, \tau)=0$. The mathematical description of the $\Sigma$ - surface is obtained by eliminating $\tau$ between $f=0$ and $g=0$ to get Eq.(14). The $\Sigma$ - surface is easily visualizable and constructible in space. Since $(\boldsymbol{x}, t)$ is fixed, $g\left(\boldsymbol{y}, \tau_{0} ; \boldsymbol{x}, t\right)=0$ is a sphere for a fixed value of source time $\tau_{0}$. The surface $f\left(\boldsymbol{y}, \tau_{0}\right)=0$ describes the frozen position of the moving body or the Kirchhoff surface at the source time $\tau_{0}$. We vary source time from $-\infty$ to $\tau=t$ and the locus of the curves of intersection of $f=0$ and $g=0$ forms a surface in space which is the $\Sigma$ - surface for the space-time observer variables $(\boldsymbol{x}, t)$. The curve of the intersection of the surfaces $f=0$ and $g=0$ is called the $\Gamma$-curve in our previous publications on rotating blade noise $[15,16,23]$. Figure 2 shows the collapsing sphere at a fixed source time intersecting a helicopter blade which is assumed to be flat. Figure 3 shows the construction of the $\Sigma$-surface for only one flat blade of a helicopter rotor in forward flight. The $\Sigma$-surface is obtained dynamically by finding the $\Gamma$-curve for several source times. Note that the $\Sigma$ - surface is swept back for the rectangular blade planform and lies in the rotor plane. It is flat, i.e. its mean curvature is zero, because we assumed a flat blade. In general, the surface $f(\boldsymbol{x}, t)=0$ is not flat and the mean curvature $H_{F}$ of its $\Sigma$ - surface is not zero. In the next section we will derive the mathematical expression for $H_{F}$.

\subsection{The Derivation of the Mean Curvature of the $\Sigma$-Surface}

We use classical differential geometry [12-14] to derive the required mean curvature of the $\Sigma$-surface. It is crucial to select appropriate variables in deriving this result for two reasons. First, as will be seen below, the algebraic manipulations are complicated and the selection of appropriate variables makes these manipulations manageable. Second, we want to give geometric 
interpretation to many of the terms in the analytic expression of the mean curvature. This requirement actually allows us to write the expression for the mean curvature in the most compact form. We mention here that, since the equation describing the $\Sigma$-surface is $F(\boldsymbol{y} ; \boldsymbol{x}, t)=0$, theoretically one should be able to get the mean curvature $H_{F}$ from this equation. Such an approach, however, appears impractical because it does not satisfy the above two requirements. First, the algebraic manipulations are intractable and the final expression for the mean curvature is very long and complicated. This complexity has undesirable impact on computer code development and efficiency. Second, we get many terms whose geometric interpretation is unclear. We, thus, looked for variables which would make the derivation of the mean curvature more direct and which would satisfy the above requirements.

\subsection{The Selection of Variables}

We describe the surface $f(\boldsymbol{y}, \tau)=0$ by the time dependent position vector $\boldsymbol{y}\left(u^{1}, u^{2}, \tau\right)$. Here, the variables $\left(u^{1}, u^{2}\right)$ are the Gaussian coordinates on the surface $f(\boldsymbol{y}, \tau)=0$ at the time $\tau$. The $\Sigma$-surface is described by the position vector:

$$
\boldsymbol{y}^{\prime}\left(u^{1}, u^{2} ; \boldsymbol{x}, t\right)=\boldsymbol{y}\left[u^{1}, u^{2}, \psi\left(u^{1}, u^{2} ; \boldsymbol{x}, t\right)\right]
$$

where the retarded or the emission time function $\psi\left(u^{1}, u^{2} ; \boldsymbol{x}, t\right)$ is the solution of the equation

$$
g=\psi-t+\frac{1}{c}\left|\boldsymbol{x}-\boldsymbol{y}\left(u^{1}, u^{2}, \psi\right)\right|=0
$$

Note that $\boldsymbol{y}, \boldsymbol{y}^{\prime}$ and $\psi$ are functions whose dependence on variables are clearly specified. Here the function $\psi$ gives the emission time of the point with Gaussian coordinates $\left(u^{1}, u^{2}\right)$ on the surface $f=0$. Therefore, $\boldsymbol{y}^{\prime}$ lies on the $\Sigma$-surface. We have implicitly defined the dependence of the position vector $\boldsymbol{y}^{\prime}$ on $\boldsymbol{y}$ through Eqs.(17) and (18). As will be seen below, this is sufficient to give us the result that we are looking for. We remind the readers that in all the analysis in this section, the observer variables $(\boldsymbol{x}, t)$ are kept fixed. 
Let $\boldsymbol{R}\left(u^{1}, u^{2}\right)$ be a surface in the three dimensional space. Denote the coefficients of the first and the second fundamental forms of this surface by $g_{i j}$ and $\mathrm{b}_{i j}$, respectively. We define the matrices $G$ and $\mathrm{G}^{-1}$ as follows:

$$
G=\left[\begin{array}{ll}
g_{11} & g_{12} \\
g_{21} & g_{22}
\end{array}\right], \quad G^{-1}=\left[\begin{array}{ll}
g^{11} & g^{12} \\
g^{21} & g^{22}
\end{array}\right]
$$

where $G^{-1}$ is the inverse of $G$. The mean curvature of the surface is given by the relation:

$$
H=\frac{1}{2} b_{i}^{i}=\frac{1}{2}\left(b_{1}^{1}+b_{2}^{2}\right)
$$

where $b_{j}^{i}=g^{i k} b_{k j}$. We have used the summation convention on repeated index here. Let $g_{(2)}=\operatorname{det}(\mathrm{G})$, then the element of the surface area is given by

$$
d S=\sqrt{g_{(2)}} d u^{1} d u^{2}
$$

The above results are in books on classical differential geometry [12-14]. We have given a summary of the useful differential geometric results in reference [16]. Below, we use Eq.(20) to derive the mean curvature of the $\Sigma$ - surface.

Remark: We use primed symbols to denote the geometric parameters of the $\Sigma$ - surface whereas the unprimed symbols are reserved for the parameters on the surface $f=0$.

\subsection{Coefficients of the First Fundamental Form of the $\Sigma$-Surface}

Let $\left(\boldsymbol{y}_{1}, \boldsymbol{y}_{2}\right)$ and $\left(\boldsymbol{y}_{1}^{\prime}, \boldsymbol{y}_{2}^{\prime}\right)$ be the natural basis vectors for the surfaces $f=0$ and the $\Sigma$ - surface $F=0$. These are defined by the relations: 


$$
\boldsymbol{y}_{i}=\frac{\partial \boldsymbol{y}}{\partial u^{i}} \quad, \quad \mathbf{y}_{i}^{\prime}=\frac{\partial \boldsymbol{y}^{\prime}}{\partial u^{i}}
$$

From Eq.(17), we find

$$
\boldsymbol{y}_{i}^{\prime}=\boldsymbol{y}_{i}+\frac{\partial \boldsymbol{y}}{\partial \tau} \frac{\partial \psi}{\partial u^{i}}
$$

From Eq.(18), we get

$$
\frac{\partial \psi}{\partial u^{i}}=\frac{\hat{r}_{i}}{c\left(1-M_{r}\right)}
$$

where $\hat{r}_{i}=\hat{\boldsymbol{r}} \cdot \mathbf{y}_{i}, \hat{\boldsymbol{r}}=|\boldsymbol{x}-\boldsymbol{y}| / r$ and $M_{r}=\boldsymbol{M} . \hat{\boldsymbol{r}}$. Here $\boldsymbol{M}$ is the local Mach number of the surface $f=0$ given by the following relation

$$
\boldsymbol{M}=\frac{1}{c} \frac{\partial y}{\partial \tau}
$$

Note that this vector is unambiguously defined even for a deforming surface. Using Eqs.(24) and (25) in Eq.(23), we get

$$
\boldsymbol{y}_{i}^{\prime}=\boldsymbol{y}_{i}+\frac{\hat{r}_{i}}{1-M_{r}} \boldsymbol{M}
$$

From this relation, we construct the coefficients of the first fundamental form of the $\Sigma$ - surface in terms of known quantities on the surface $f=0$ as follows:

$$
g_{i j}^{\prime}=g_{i j}+\frac{M_{i} \hat{r}_{j}+M_{j} \hat{r}_{i}}{1-M_{r}}+\frac{M^{2} \hat{r}_{i} \hat{r}_{j}}{\left(1-M_{r}\right)^{2}}
$$

where $g_{i j}=y_{i} \cdot y_{j}$ is the coefficients of the first fundamental form of the surface $f=0, M_{i}=\boldsymbol{M} \cdot \boldsymbol{y}_{i}$ is the covarient component of $\boldsymbol{M}$ with respect to the natural basis vectors on the surface $f=0$, and $M=|\boldsymbol{M}|$. Note that $g_{i j}^{\prime}$ 
depends on the geometric parameters of the surface $f=0$, the kinematic parameters as well as the radiation direction $\hat{r}_{i}$.

From Eq.(27), we find the determinant of the coefficients of the first fundamental form of the $\Sigma$-surface. The derivation of this result involves much algebraic manipulations. The final result, however, is simple and it is

$$
g_{(2)}^{\prime}=\left(\frac{\Lambda}{1-M_{r}}\right)^{2} g_{(2)}
$$

where $\Lambda$ is given by Eq.(12) and $g_{(2)}$ is the determinant of the first fundamental form of the surface $f=0$. Now we use this equation to relate the element of surface areas $d \Sigma$ and $d S$ of the $\Sigma$-surface and the surface $f=0$, respectively. This relation is obtained from the determinants of the coefficients of the first fundamental forms on these surfaces and using Eq.(21). It is

$$
d \Sigma=\sqrt{g_{(2)}^{\prime}} d u^{1} d u^{2}=\frac{\Lambda}{\left|1-M_{r}\right|} \sqrt{g_{(2)}} d u^{1} d u^{2}=\frac{\Lambda}{\left|1-M_{r}\right|} d S
$$

This very useful result was originally obtained by the first author using a geometrical method [23].

We can now find the elements $g^{i j}$ of the inverse of the matrix $G^{\prime}=\left[g_{i j}^{\prime}\right]$. These are defined as follows

$$
g^{\prime 11}=\frac{g_{22}^{\prime}}{g_{(2)}^{\prime}}, \quad g^{g^{12}}=g^{\prime^{21}}=-\frac{g_{12}^{\prime}}{g_{(2)}^{\prime}}, \quad g^{{ }^{\prime 2}}=\frac{g_{11}^{\prime}}{g_{(2)}^{\prime}}
$$

We have given $g_{i j}^{\prime}$ and $g_{(2)}^{\prime}$ above in Eqs.(27) and (28). We introduce two new vectors tangent to the surface $f=0$ :

$$
\gamma=n \times \hat{r}, \quad \lambda=n \times M
$$


in order to write $g^{\prime i j}$ in contravariant tensor form. We note that the contravariant components of the vectors $\gamma$ and $\lambda$ are given by the relations

$$
\begin{array}{ll}
\gamma^{1}=-\frac{\hat{r}_{2}}{\sqrt{g_{(2)}}}, & \gamma^{2}=\frac{\hat{r}_{1}}{\sqrt{g_{(2)}}}, \\
\lambda^{1}=-\frac{M_{2}}{\sqrt{g_{(2)}}}, & \lambda^{2}=\frac{M_{1}}{\sqrt{g_{(2)}}},
\end{array}
$$

respectively. We can then write $g^{i j}$ as follows:

$$
g^{i j}=\frac{\left(1-M_{r}\right)^{2}}{\Lambda^{2}} g^{i j}+\frac{1-M_{r}}{\Lambda^{2}}\left(\gamma^{i} \lambda^{j}+\gamma^{j} \lambda^{i}\right)+\frac{M^{2}}{\Lambda^{2}} \gamma^{i} \gamma^{j}
$$

We will use this result in the next subsection.

Remark: We clarify an important point about notation here. What is the meaning of the parameters of the surface $f=0$ on the $\Sigma$-surface in the above equations? The $\Sigma$-surface is given by $F(\boldsymbol{y} ; \boldsymbol{x}, t)=0$ where the observer variables $(\boldsymbol{x}, t)$ are fixed. An emission time is associated with each point $\mathbf{y}$ on this surface. This emission time is given by $t-|\boldsymbol{x}-\boldsymbol{y}| / c$. At this instant, the surface $f=0$ intersects the $\Sigma$ - surface along a curve which we called the $\Gamma$-curve (see Figs. 2 and 3). All parameters of the surface $f=0$ at the point $\mathbf{y}$ on the $\Sigma$-surface are calculated at the emission time of the point $\mathbf{y}$. This means that a parameter such as $M_{r}$ in Eq.(29) is evaluated as follows:

$$
M_{r}(\boldsymbol{y} ; x, t) \equiv \boldsymbol{M}\left(\boldsymbol{y}, t-\frac{|\boldsymbol{x}-\boldsymbol{y}|}{c}\right) . \hat{\boldsymbol{r}}
$$

where $\boldsymbol{M}$ and $\hat{\boldsymbol{r}}=(\boldsymbol{x}-\boldsymbol{y}) /|\boldsymbol{x}-\boldsymbol{y}|$ are calculated from the surface $f=0$ but precisely on the $\Gamma$-curve it shares with the $\Sigma$-surface. Note that all the variables $(\boldsymbol{y}, \boldsymbol{x}, t)$ on the right of this equation are independent variables and 
there is no ambiguity in the interpretation of the dependent variables as we have written them here. As explained below, differentiation of the dependent variables with respect to variables $\left(u^{1}, u^{2}\right)$, however, needs more care.

\subsection{Coefficients of the Second Fundamental Form of the $\Sigma$-Surface}

The coefficients of the second fundamental forms of the surface $f=0$ and the $\Sigma$ - surface are defined by the relations:

$$
b_{i j}=\boldsymbol{n} \cdot \frac{\partial^{2} \boldsymbol{y}}{\partial u^{i} \partial u^{j}}=\boldsymbol{n} \cdot \boldsymbol{y}_{i j}, b_{i j}^{\prime}=N \cdot{\frac{\partial^{2} \boldsymbol{y}^{\prime}}{\partial u^{i} \partial u^{j}}}^{\prime}=N \cdot \boldsymbol{y}_{i j}^{\prime}
$$

where $N$ is the unit normal to the $\Sigma$ - surface which is given by

$$
N=\frac{\nabla F}{|\nabla F|}=\frac{\boldsymbol{n}-M_{n} \hat{\boldsymbol{r}}}{\Lambda}
$$

The subscripts of $\mathbf{y}$ and $\boldsymbol{y}^{\prime}$ denote differentiation with respect to variables $\left(u^{1}, u^{2}\right)$. As will be shown below, the derivation of the analytic expression for $b_{i j}^{\prime}$ requires considerable amount of algebraic manipulations. The reason is the dependence of the emission time on the variables $\left(u^{1}, u^{2}\right)$. Thus, for any independent variable defined on the $\Sigma$ - surface, e.g. $\mathbf{n}$ above, we have

$$
\left.\frac{\partial \boldsymbol{n}}{\partial u^{i}}\right|_{\Sigma}=\frac{\partial}{\partial u^{i}} \boldsymbol{n}\left(u^{1}, u^{2}, \psi\left(u^{1}, u^{2} ; \boldsymbol{x}, t\right)\right)=\frac{\partial}{\partial u^{i}} \boldsymbol{n}\left(u^{1}, u^{2}, \psi\right)+\frac{\partial \boldsymbol{n}}{\partial \tau} \frac{\partial \psi}{\partial u^{i}} \text { (EQ 38) }
$$

where the emission time function $\psi$ is given by Eq.(18). Here, on the right of the second equality sign, we have taken the unit normal $\boldsymbol{n}$ as a function of variables $\left(u^{1}, u^{2}, \tau\right)$. Note that $\boldsymbol{n}\left(u^{1}, u^{2}, \tau\right)$ and $\boldsymbol{n}\left(u^{1}, u^{2}, \psi\left(u^{1}, u^{2} ; \boldsymbol{x}, t\right)\right)$ are two different functions- the former is defined on the surface $f=0$ and the latter on the $\Sigma$ - surface. Strictly speaking, we should use different symbols for these functions but this is not done in practice because it leads to pro- 
fusion of symbols which can be annoying. We should warn the readers that this practice, common as it is, can lead to confusion at times in wave propagation problems. For this reason, we have made an exception to the usual practice for one symbol that appears very often in our work. We introduced the symbol $\psi$ for the retarded time function in place of which we could have used the same symbol as the source time $\tau$. We have reserved $\tau$ for an independent variable.

To reduce the risk of confusion in interpreting a partial differentiation with respect to $\left(u^{1}, u^{2}\right)$, we specifically indicate this on the $\Sigma$ - surface as follows $\partial /\left.\partial u^{i}\right|_{\Sigma}$. This means that the independent variable is a function of $\left(u^{1}, u^{2} ; \boldsymbol{x}, t\right)$. However, we use the notation $\partial / \partial u^{i}$ for partial differentiation of an independent variable which is a function of $\left(u^{1}, u^{2}, \tau\right)$ on the surface $f=0$. There is no confusion for the partial derivative $\partial / \partial \tau$ which applies only to the independent variables on $f=0$. Note that on the $\Sigma$ - surface, differentiation with respect to the source time is meaningless since the independent variables on this surface are not functions of $\tau$.

By taking the derivative with respect to the variable $u^{j}$ of both sides of Eq. (23) and after much algebraic manipulations and simplifications, we get

$$
\begin{aligned}
& \boldsymbol{y}_{i j}{ }^{\prime}=\boldsymbol{y}_{i j}-\frac{g^{\prime}{ }_{i j} \boldsymbol{M}}{r\left(1-M_{r}\right)}+\frac{1}{1-M_{r}}\left(\hat{r}_{j} \frac{\partial \boldsymbol{M}}{\partial u^{i}}+\hat{r}_{i} \frac{\partial \boldsymbol{M}}{\partial u^{j}}\right) \\
& +\frac{\boldsymbol{M}}{1-M_{r}}\left(\Gamma_{i j}^{k} r_{k}+b_{i j} \cos \theta\right) \\
& +\frac{\hat{r}_{i} \hat{r}_{j} \dot{\boldsymbol{M}}}{c\left(1-M_{r}\right)^{2}}+\frac{\boldsymbol{M}}{\left(1-M_{r}\right)^{2}}\left(r_{i} \hat{\boldsymbol{r}} \cdot \frac{\partial \boldsymbol{M}}{\partial u^{j}}+r_{j} \hat{\boldsymbol{r}} \cdot \frac{\partial \boldsymbol{M}}{\partial u^{i}}\right) \\
& +\frac{\dot{M}_{r} \hat{r}_{i} \hat{r}_{j} \boldsymbol{M}}{c\left(1-M_{r}\right)^{3}}+\frac{\hat{r}_{i} \hat{r}_{j} \boldsymbol{M}}{r\left(1-M_{r}\right)^{3}}
\end{aligned}
$$


Here $\Gamma_{i j}^{k}$ is the Christoffel symbol of the second kind, $\theta$ is the angle between $\boldsymbol{n}$ and the radiation direction $\hat{\boldsymbol{r}}$, and $\dot{M}_{r}=\hat{\boldsymbol{r}} \cdot \dot{\boldsymbol{M}}$. We have summed some terms on the right sides to get $g_{i j}^{\prime}$ in the numerator of the second term because in obtaining the mean curvature of the $\Sigma$ - surface, we must multiply $b^{\prime}{ }_{i j}$ with $g^{\prime i j}$ as will be seen later. Therefore, this summation reduces some algebraic manipulations.

\subsection{The Mean Curvature of the $\Sigma$-Surface for a Rigid Moving Surface}

So far we have not assumed that the surface $f=0$ is rigid. All the above results are valid for a deformable surface. Now, we will make the assumption of rigidity of the surface. In applications that we have in mind, the surface $f=0$ is always rigid, e.g., the surface of a propeller or rotor blade. The Mach vector distribution of the surface can be written as $\boldsymbol{M}=\left(v_{0}(\tau)+\omega \times \eta\right) / c$ where $v_{0}(\tau)$ is the velocity of a reference point on the surface, $\eta$ is the position vector of a point on the surface from the reference point, and $\omega$ is the angular velocity of the surface. We note that when $\tau$ is fixed, we can assume that $\boldsymbol{y}$ and $\eta$ coincide and, thus, we get $\partial \boldsymbol{M} / \partial u^{i}=\omega \times \boldsymbol{y}_{i} / c$. Using the definition of the second fundamental form, Eq. (36), we obtain from Eqs. (37) and (39):

$$
\begin{aligned}
& b_{i j}^{\prime}=\frac{b_{i j}}{\Lambda}+\frac{\hat{r}_{i} q_{j}+\hat{r}_{j} q_{i}}{\Lambda\left(1-M_{r}\right)}+\frac{\hat{r}_{i} \hat{r}_{j} \dot{M}_{n}}{c \Lambda\left(1-M_{r}\right)^{2}} \\
& -\frac{M_{n} g_{i j}^{\prime}}{r \Lambda}+\frac{\hat{r}_{i} \hat{r}_{j} M_{n}}{r \Lambda\left(1-M_{r}\right)^{2}}
\end{aligned}
$$

where we have defined

$$
\boldsymbol{q}=\frac{1}{c} n \times \omega
$$


and $\Lambda$ is given by Eq. (12). We are now ready to find the mean curvature of the $\Sigma$ - surface for a rigid moving surface.

From the definition of the mean curvature, Eq. (20), using primed variables for the $\Sigma$ - surface, we obtain the following expression for $H_{F}$ :

$$
\begin{aligned}
H_{F}= & -\frac{M_{n}}{r \Lambda}\left(1-\frac{\sin ^{2} \theta}{2 \Lambda^{2}}\right)+\frac{\left(1-M_{r}\right)^{2}}{\Lambda^{3}} H_{f} \\
& +\frac{1-M_{r}}{\Lambda^{3}}\left(\hat{\boldsymbol{r}} \cdot \boldsymbol{q}+\kappa_{1} \tilde{\lambda}^{1} \tilde{\gamma}^{1}+\kappa_{2} \tilde{\lambda}^{2} \tilde{\gamma}^{2}\right) \\
& +\frac{\sin ^{2} \theta}{2 \Lambda^{3}}\left(\frac{1}{c} \dot{M}_{n}+M^{2} \kappa_{\gamma}\right)+\frac{1}{\Lambda^{3}}(\boldsymbol{\gamma} \cdot \boldsymbol{q})(\boldsymbol{\lambda} \cdot \hat{\boldsymbol{r}})
\end{aligned}
$$

Here we have used the following definitions of the symbols:

$$
\begin{array}{ll}
\Lambda & =\left(1+M_{n}^{2}-2 M_{n} \cos \theta\right)^{1 / 2} \\
\cos \theta & =\boldsymbol{n} \cdot \hat{\boldsymbol{r}} \\
\hat{\boldsymbol{r}} & =(\boldsymbol{x}-\boldsymbol{y}) / r \text { unit radiation vector } \\
\boldsymbol{n} & =\nabla f \text { (unit outward normal to } f=0) \\
\boldsymbol{M} & =\partial \boldsymbol{y} / c \partial \tau, \quad M=|\boldsymbol{M}|, \quad \dot{M}_{n}=\dot{\boldsymbol{M}} \cdot \boldsymbol{n} \\
H_{f} & =\text { local mean curvature of } f=0 \\
\kappa_{1} \text { and } \kappa_{2}=\text { local principal curvatures of } f=0 \\
\kappa_{\gamma} & =\text { local normal curvature of } f=0 \text { along } \gamma \\
\gamma & =\boldsymbol{n} \times \hat{\boldsymbol{r}} \\
\lambda & =\boldsymbol{n} \times \boldsymbol{M} \\
\boldsymbol{q} & =\boldsymbol{n} \times \omega / c \\
\omega & =\text { angular velocity of } f=0
\end{array}
$$

$\left(\tilde{\gamma}^{1}, \tilde{\gamma}^{2}\right)$ and $\left(\tilde{\lambda}^{1}, \tilde{\lambda}^{2}\right)$ are the components of $\gamma$ and $\lambda$ (see Eq.(31)) in principal directions with respect to unit basis vectors

Equation (42) is the main result of this paper. This result was derived and announced by the first author in 1996 [16] but the derivation has not been published. The second author independently verified the final result through 
a slightly different approach. The extension to the deformable case was also carried out by the second author which will now be presented.

\subsection{The Mean Curvature of the $\Sigma$-Surface for a Deformable Moving Surface}

If we assume the surface $f=0$ is deformable, then we get a slightly more complicated expression for the mean curvature of the $\Sigma$ - surface . In the derivation of the mean curvature formula, Eq.(42), the rigidity hypothesis was first introduced during the computation of the second fundamental form, Eq.(40). In order to avoid this restriction, we begin by rewriting Eq.(39) as follows:

$$
\begin{gathered}
\boldsymbol{y}_{i j}^{\prime}=T\left(\boldsymbol{y}_{i j}+c \frac{\partial \psi}{\partial u^{i}} \frac{\partial \boldsymbol{M}}{\partial u^{j}}+c \frac{\partial \psi}{\partial u^{j}} \frac{\partial \boldsymbol{M}}{\partial u^{i}}\right) \\
+\frac{\boldsymbol{M}}{r\left(1-M_{r}\right)}\left(-g_{i j}^{\prime}+c^{2} \frac{\partial \psi}{\partial u^{i}} \frac{\partial \psi}{\partial u^{j}}\right)
\end{gathered}
$$

Here $T$ is a linear operator defined by

$$
T(\boldsymbol{V})=\boldsymbol{V}+\frac{\hat{\boldsymbol{r}} \cdot \boldsymbol{V}}{1-M_{r}} \boldsymbol{M}
$$

for a given vector $\boldsymbol{V}$. Next we compute $b_{i j}^{\prime}=\boldsymbol{y}_{i j}^{\prime} \cdot \boldsymbol{N}$. To do this, note that we can show $T(\boldsymbol{V}) \cdot \boldsymbol{N}=\boldsymbol{V} \cdot \boldsymbol{n} / \Lambda$. From Eq. (43), we get

$$
\begin{aligned}
& b_{i j}^{\prime}=\frac{1}{\Lambda}\left[b_{i j}+\frac{1}{1-M_{r}}\left(\hat{r}_{i} \frac{\partial \boldsymbol{M}}{\partial u^{j}} \cdot \boldsymbol{n}+\hat{r}_{j} \frac{\partial \boldsymbol{M}}{\partial u^{i}} \cdot \boldsymbol{n}\right)\right. \\
& \left.+\frac{\hat{r}_{i} \hat{r}_{j}}{c\left(1-M_{r}\right)^{2}} \frac{\partial \boldsymbol{M}}{\partial \tau} \cdot \boldsymbol{n}+\frac{M_{n}}{r}\left(-g_{i j}^{\prime}+\frac{\hat{r}_{i} \hat{r}_{j}}{\left(1-M_{r}\right)^{2}}\right)\right]
\end{aligned}
$$

Considerable algebra is now involved to get the mean curvature of the $\Sigma$ - surface. Taking advantage of the symmetry of the first and the second fundamental forms of the surface $f=0$ and the orthogonality of the vectors $\hat{\boldsymbol{r}}$ and $\gamma$ yield: 


$$
\begin{aligned}
& H_{F}=-\frac{M_{n}}{r \Lambda}+\frac{1}{2 \Lambda^{3}}\left[\sin ^{2} \theta\left(\dot{M}_{n}+\frac{M_{n}}{r}\right)\right. \\
& +2 H_{f}\left(1-M_{r}\right)^{2}+2\left(1-M_{r}\right) L(\gamma, \lambda)+M^{2} L(\gamma, \gamma) \\
& \left.-\frac{1}{c} \frac{\partial \boldsymbol{n}}{\partial \tau} \cdot\left[2\left(1-M_{r}\right) \hat{\boldsymbol{r}}_{t}+2\left(\lambda \cdot \hat{\boldsymbol{r}}_{t}\right) \gamma+\sin ^{2} \theta M\right]\right]
\end{aligned}
$$

Here the operator $\mathbf{L}$ is based on the coefficients of the second fundamental form defined as $L(\boldsymbol{U}, \boldsymbol{V})=b_{i j} U^{i} V^{j}$ and the vector $\hat{\boldsymbol{r}}_{t}$ is the projection of the unit radiation vector $\hat{\boldsymbol{r}}$ on the local tangent plane to the surface $f=0$. Note that $\left|\hat{r}_{t}\right|=\sin \theta$.

For a deformable surface, a new problem appears which must be addressed carefully. Note that for the case of a rigid surface, all quantities on the right side of Eq. (42), or equivalently Eq. (46), all surface coordinates are independent and all the velocities are unambiguously defined. In the case of a deformable surface, we must show that if two surface Gaussian coordinate systems slide with respect to each other, then the mean curvature of the $\Sigma$ - surface is independent of the tangential Mach numbers of the two coordinate systems. The terms of the mean curvature expression for the $\Sigma$ - surface of a deformable surface can be written in such a way that the tangential Mach number of the coordinate system is taken out. In order to accomplish this, we decompose $\mathbf{M}$ into its normal and tangential components on the surface $f=0$ denoted $\boldsymbol{M}_{n}$ and $\boldsymbol{M}_{t}$, respectively. We then separate the mean curvature expression into pieces that involve these normal and tangential components separately. We also remove the explicit dependence on the vectors $\gamma$ and $\lambda$ by using the identity

$$
L\left(\boldsymbol{M}_{t}, \hat{\boldsymbol{r}}_{t}\right)+L(\gamma, \lambda)=2 H_{f} \boldsymbol{M}_{t} \cdot \hat{\boldsymbol{r}}_{t}
$$

This allows the mean curvature of the $\Sigma$ - surface to be written as 


$$
\begin{aligned}
& H_{F}=-\frac{M_{n}}{r \Lambda}+\frac{H_{f}}{\Lambda}+\frac{1}{2 \Lambda^{3}}\left[\operatorname { s i n } ^ { 2 } \theta \left(\frac{M_{n}}{r}-M_{n}^{2} \kappa_{r}+\dot{M}_{n}\right.\right. \\
& \left.+\frac{1}{c} \boldsymbol{M}_{t} \cdot \frac{\partial \boldsymbol{n}}{\partial \tau}+M_{t}^{2} \kappa_{M}\right) \\
& \left.-2\left(1-M_{n} \cos \theta\right)\left(\frac{1}{c} \hat{\boldsymbol{r}} \cdot \frac{\partial \boldsymbol{n}}{\partial \tau}+L\left(\hat{\boldsymbol{r}}_{t}, \boldsymbol{M}_{t}\right)\right)\right]
\end{aligned}
$$

where $\kappa_{r}$ and $\kappa_{M}$ are the normal curvatures of the surface $f=0$ in the directions of $\hat{\boldsymbol{r}}_{t}$ and $\boldsymbol{M}_{t}$, respectively. We have used the fact that $L(\boldsymbol{V}, \boldsymbol{V})=|\boldsymbol{V}|^{2} \kappa_{V}$, where $\kappa_{V}$ is the normal curvature of $f=0$ in the direction of the tangential vector $\boldsymbol{V}$.

In Eq. (48), everything is clearly independent of frame of reference except, apparently, for the expressions $\frac{1}{c} \hat{\boldsymbol{r}}_{t} \cdot \frac{\partial \boldsymbol{n}}{\partial \tau}+L\left(\hat{\boldsymbol{r}}_{t}, \boldsymbol{M}_{t}\right) \quad$ and $\dot{M}_{n}+\frac{1}{c} M_{t} \cdot \frac{\partial \boldsymbol{n}}{\partial \tau}+M_{t}^{2} \kappa_{M}$.It turns out that these expressions are also independent of frame of reference. In fact, it can be shown that

$$
\frac{1}{c} \hat{\boldsymbol{r}}_{t} \cdot \frac{\partial \boldsymbol{n}}{\partial \tau}+L\left(\hat{\boldsymbol{r}}_{t}, \boldsymbol{M}_{t}\right)=\frac{1}{c} \hat{\boldsymbol{r}}_{t} \cdot \nabla f_{\tau}
$$

and

$$
\dot{M}_{n}+\frac{1}{c} \boldsymbol{M}_{t} \cdot \frac{\partial \boldsymbol{n}}{\partial \tau}+M_{t}^{2} \kappa_{M}=-\frac{1}{c} f_{\tau \tau}+\frac{1}{c} f \tau
$$

Although Eqs. (49) and (50) show that the mean curvature expression Eq (48) is independent of the coordinate system chosen, Eq. (48) as it stands might be more suitable for use in practice.

Now that we have derived the analytic expression for the mean curvature of the $\Sigma$-surface, we can write the solution of the FW-H equation and the Kirchhoff formula for a supersonically moving surface in a very compact way using Eqs.(11) and (13) in the solution of the wave Eqs.(4) and (8). We 
feel that by relating the mean curvature of the $\Sigma$ - surface to the geometric and kinematic parameters of the surface $f=0$, we have derived useful formulas for applications in aeroacoustics.

\subsection{Discussion and Concluding Remarks}

The mean curvature of the $\Sigma$ - surface is a very complicated expression and there is little hope that for moving surfaces of engineering interest one can obtain an analytic result given the geometry and the history of the motion of the moving surface $f=0$. A simple but not sufficient test of validity of $H_{F}$ is that for a stationary surface, one should get $H_{F}=H_{f}$. This can be easily established from Eq. (42). However, numerical test of this result is possible for a given smooth surface in rectilinear and rotating motion. Note that the mean curvature of the collapsing sphere is $1 / r$. As seen from Eq. (42), only the first term of the mean curvature of the $\Sigma$-surface depends on the mean curvature of the collapsing sphere.

One of the main reasons for studying the mean curvature of the $\Sigma$ - surface has been to explain the complexity and the appearance of normal curvatures of the surface $f=0$ in many directions in Formulation 3 of Farassat used for supersonic propeller noise prediction [11]. It is now clear that Formulation 3 can be written in a much more compact form using the mean curvature of the $\Sigma$ - surface. The procedure for the derivation of this compact formula has been outlined in Section 2 of this paper. Such a formula will simplify coding for noise prediction by calculating $H_{F}$ in a separate subroutine. We have made further progress in finding a very simple solution of Eq. (10) which does not depend on the mean curvature of the $\Sigma$ - surface [24]. We feel, however, that the solution involving the mean curvature discussed here is fundamental and it will lead to further understanding of noise generation from moving surfaces.

There is one important point that must be mentioned in connection with the solution of the wave equation of Eq. (10). When the collapsing sphere leaves the surface $f=0$ at a point where $M_{n}=1$, then one gets $\Lambda=0$ in the denominator of the integrand of the solution of Eq. (10) given by Eq. (13). This can lead to infinite singularity in the acoustic pressure $p^{\prime}$. Based on a 
recent study by Farassat, Brentner and Dunn [24], it can be shown that in Eq. (13), there is a cancellation of the highest order of $1 / \Lambda$ singularity between the first and the second terms in the integrands on the right side of the equation. Because of the particular structure of $\underset{\sim}{q}(\boldsymbol{y}, \tau)$ in the governing equation of the Kirchhoff formula [24], the acoustic pressure calculated from the Kirchhoff formula is finite for all time. Similar situation exists for the FW-H equation if we add the surface terms coming from the quadrupole source term to the thickness and loading terms [24]. However, noise calculation based on the thickness and loading terms alone for supersonic blades with blunt leading edge can result in infinite acoustic pressure.

\section{Acknowledgment}

The second author was an American Society for Engineering Education (ASEE) Summer Faculty Fellow at NASA Langley Research Center in 1997. The work reported here was performed during this period in collaboration with the first author.

\subsection{References}

1. Ffowcs Williams, J. E. and Hawkings, D.L., "Sound generation by turbulence and surfaces in arbitrary motion", Phil Trans. Roy. Soc. (London), 264A, 321-342 (1969)

2. Hawkings, D.L., "Noise generation by transonic open rotors", Westland Research Paper 599 (1979)

3. Hubbard, Harvey H. (Editor), "Aeroacoustics of Flight Vehicles - Theory and Practice", 2 volumes, Acoustical Society of America, 1995

4. Goldstein, Marvin, E., "Aeroacoustics", McGraw-Hill International Book Company, New York, 1976

5. Lyrintzis, A. S., "Review: The Use of Kirchhoff's Method in Computational Aeroacoustics”, ASME Journal of Fluids Engineering, 116, 665-676 (1994) 
6. Crighton, D. G., Dowling, A. P., Ffowcs Williams, J. E., Heckl, M and Leppington, F. G., "Modern Methods in Analytical Acoustics", Springer-Verlag, London, 1992

7. Di Francescantonio, P., "A new boundary integral formulation for the prediction of sound radiation", J. Sound and Vib., 202, 491-509 (1997)

8. Brentner, Kenneth S. and Farassat, F., "Analytical comparison of the acoustic analogy and the Kirchhoff formulation for moving surfaces, AIAA J., 36, 1379-1386 (1998)

9. Farassat, F., "Generalized functions and Kirchhoff formulas", AIAA/CEAS Paper No. 96-1705 (1996) (Available at ftp://techreports.larc.nasa.gov/pub/techreports/larc/96/NASA-aiaa-96-1705.ps.Z)

10. Farassat, F. and Myers, M. K., "Aeroacoustics of high-speed rotating blades: The mathematical aspect", 117-148. In Computational Acoustics Acoustic Propagation, D. Lee, R. Vichnevetsky, and A. R. Robinson, North-Holland, 1993

11. Farassat, F., Padula, S. L. and Dunn, M. H., "Advanced turboprop noise prediction based on recent theoretical results", J. of Sound and Vib., 119, 53-79 (1987)

12. Kreyszig, Erwin, Differential Geometry, Dover Books, 1991

13. McConnell, A. J., Applications of Tensor Analysis, Dover Books, 1957

14. Aris, R., Vectors, Tensors and the Basic Equations of Fluid Mechanics, Dover Books, 1989

15. Farassat, F., "Introduction to generalized functions with applications in aerodynamics and aeroacoustics", NASA TP-3428 (1994), Corrected copy (April 1996), (Available at ftp://techreports.larc.nasa.gov/pub/techreports/larc/94/tp3428.ps.Z)

16. Farassat, F., "The Kirchhoff formulas for moving surfaces in aeroacoustics- The subsonic and supersonic cases", NASA Technical Memorandum 110285 (1996), (Available at ftp://techreports.larc.nasa.gov/pub/techreports/larc/96/NASA-96-tm110285.ps.Z) 
17. Farassat F. and Succi, G. P., "The prediction of helicopter rotor discrete frequency noise", Vertica, 7, 309-320 (1983)

18. Brentner, Kenneth S., "Prediction of helicopter rotor discrete frequency noise- A computer program incorporating realistic blade motions and advanced acoustic formulation", NASA Technical Memorandum 87721 (1986)

19. Gelfand, I. M. and Shilov, G. E. Generalized Functions, Vol. 1, Academic Press, 1964

20. Jones, D. S., Generalized Functions, second edition, Cambridge University press, 1982

21. Courant, R., and Hilbert, D., Methods of Mathematical Physics, Vol. 2, Partial Differential Equations, Interscience Publishers, 1962.

22. Smith, M. G., Introduction to the Theory of Partial Differential Equations, Van Nostrand, 1967

23. Farassat, F., "Theory of noise generation from moving bodies with an application to helicopter rotors", NASA TR R-451 (1975), (Available at http://techreports.larc.nasa.gov/ltrs/PDF/NASA-75-trr451.pdf)

24. Farassat, F., Brentner, Kenneth S. and Dunn, M. H., "A study of supersonic surface sources- The Ffowcs Williams-Hawkings equation and the Kirchhoff formula", AIAA Paper 98-2375 (1998) (Available at http://techreports.larc.nasa.gov/ltrs/PDF/1998/aiaa/NASA-aiaa-98-2375.pdf) 


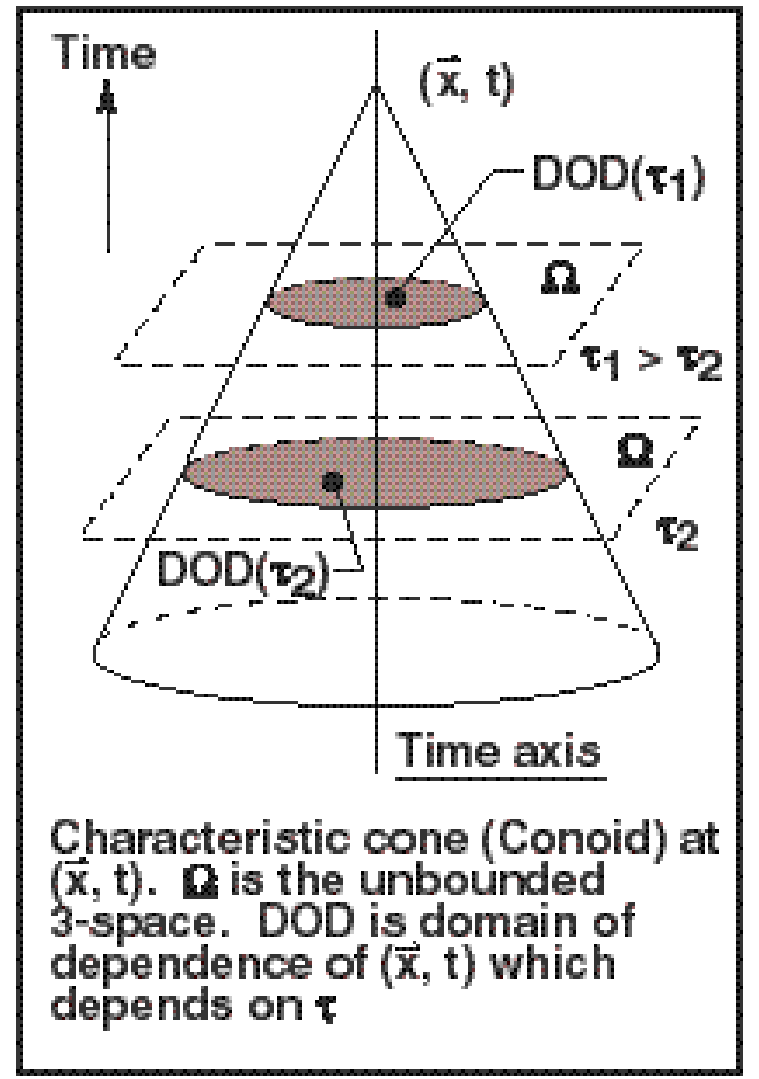

Figure 1. Schematic diagram of the characteristic cone (conoid) of the observer space time variable $(\boldsymbol{x}, t)$ and its domain of dependence (DOD). 


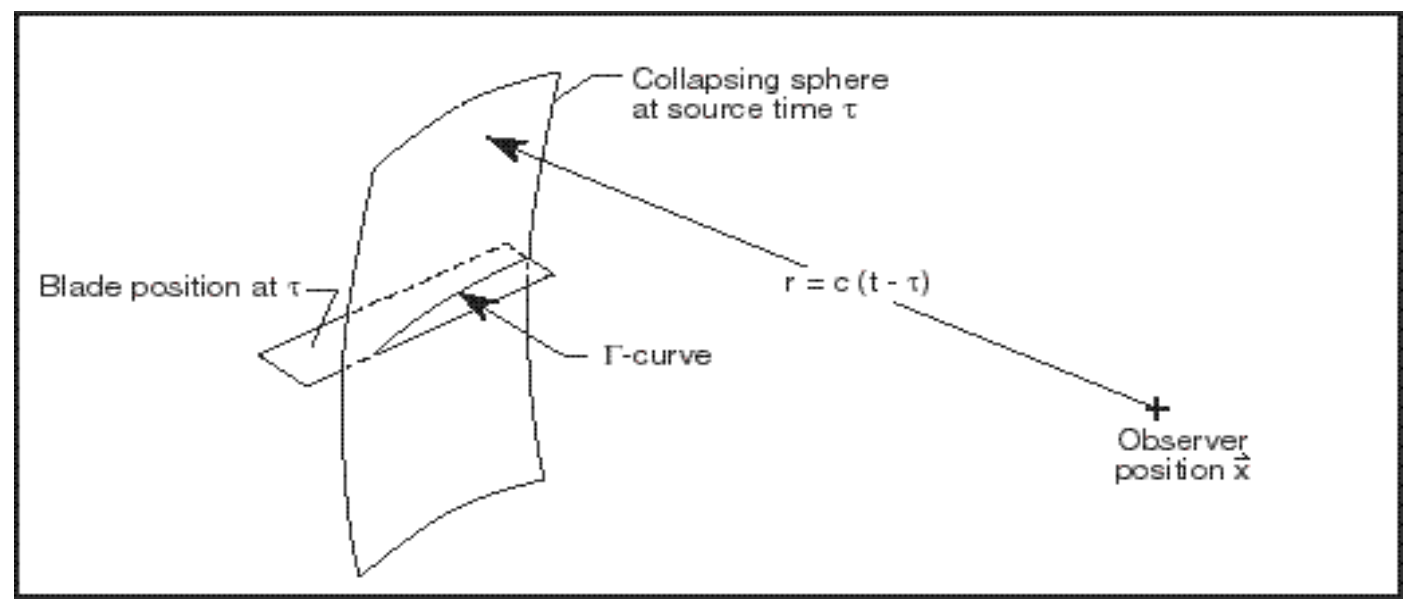

Figure 2. The collapsing sphere intersecting a rotating blade at the source time $\tau$. Note that the collapsing sphere is the intersection of the characteristic cone (conoid) of $(\boldsymbol{x}, t)$ with the three dimensional space at the time $\tau$.

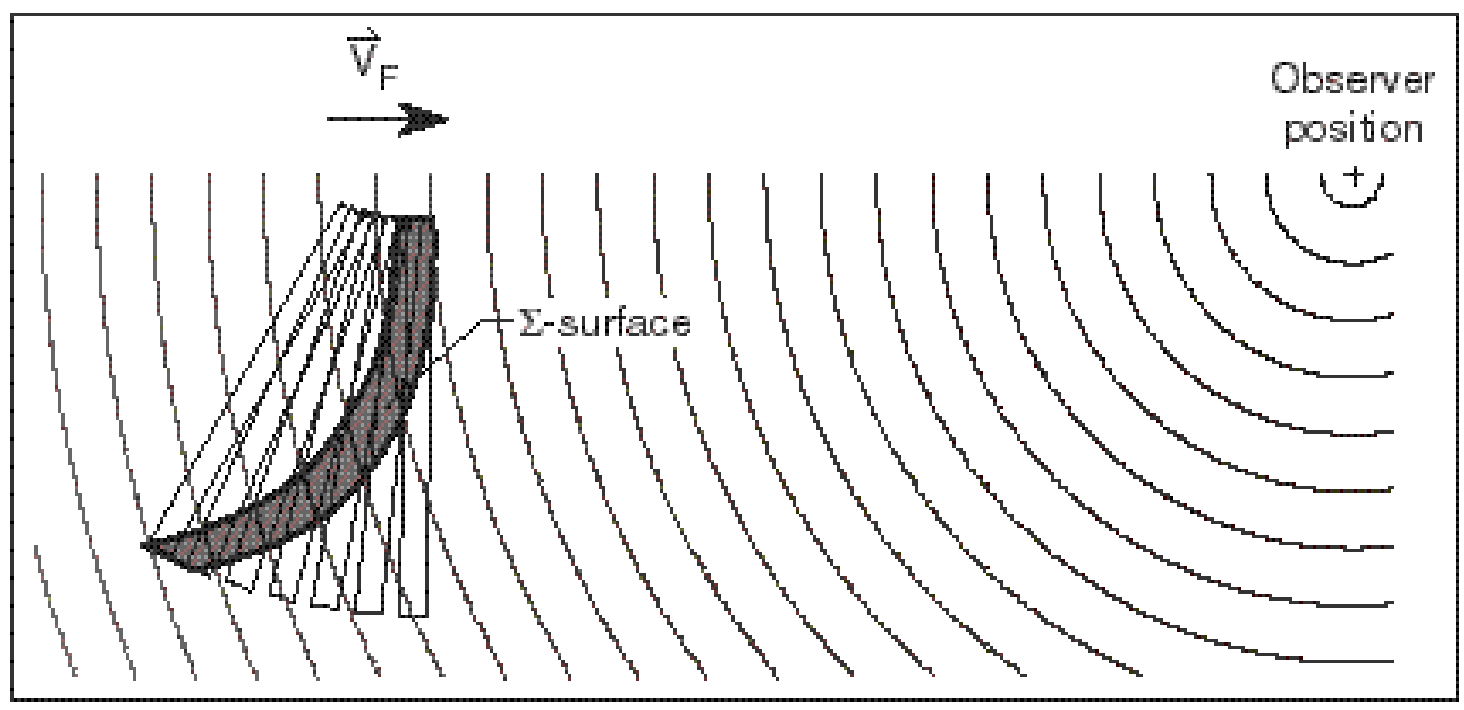

Figure 3. The construction of the $\Sigma$-surface for a flat rectangular rotor blade. The rotor is moving forward at the velocity $\boldsymbol{V}_{F}$ in such a way that all points on the rotor travel at subsonic speed relative to the speed of sound in undisturbed medium. The $\Sigma$ - surface will be more complicated and even in several pieces if part or all the blade surface travel at supersonic speed 